\title{
Using WordNet to Posit Hierarchical Structure in Levin's Verb Classes
}

\author{
Mari Broman Olsen, Bonnie J. Dorr, and David J. Clark \\ Institute for Advanced Computer Studies \\ University of Maryland \\ College Park, MD, USA 20742 \\ \{molsen, bonnie, dav\}@umiacs. umd . edu
}

\begin{abstract}
In this paper we report on experiments using WordNet synset tags to evaluate the semantic properties of the verb classes cataloged by Levin (1993). This paper represents ongoing research begun at the University of Pennsylvania (Rosenzweig and Dang, 1997; Palmer, Rosenzweig, and Dang, 1997) and the University of Maryland (Dorr and Jones, 1996b; Dorr and Jones, 1996a; Dorr and Jones, 1996c). Using WordNet sense tags to constrain the intersection of Levin classes, we avoid spurious class intersections introduced by homonymy and polysemy (run a bath, run a mile). By adding class intersections based on a single shared sense-tagged word, we minimize the impact of the non-exhaustiveness of Levin's database (Dorr and Olsen, 1996; Dorr, To appear). By examining the syntactic properties of the intersective classes, we provide a clearer picture of the relationship between WordNet/EuroWordNet and the LCS interlingua for machine translation and other NLP applications.
\end{abstract}

\section{Introduction}

WordNet is a network of basic semantic relations between English words. An effort is also underway to develop a multi-lingual EuroWordNet (Dutch, Italian, and Spanish (Calzolari et al., To appear)) with links to the English database (Miller, 1986; Miller, 1990; Miller and Fellbaum, 1991). Levin (1993) has 4183 verbs organized into a relatively flat structure: ${ }^{1}$ a list of 191 classes, derived from verb behavior with respect to certain syntactic "alternations."

Although the organizing principles of these resources are arguably orthogonal, attempts have been made to identify relations between WordNet and Levin's classes. Jones and Onyshkevych (1996), for example, report an experiment investigating the extent to which Levin's classes capture synonymy relations in WordNet. Using only the verbs from Levin, they found $38 \%$ of the synonym sets described by WordNet were wholly captured by Levin's classes. They attributed the remaining $62 \%$ to three factors, without exploring their distribution: errors and omissions in WordNet, errors and omissions in Levin, and "intrinsic orthog-

\footnotetext{
${ }^{1}$ Although Levin's book has classes and subclasses, the relation between them is not consistent. Furthermore, verbs in different classes have a subset or intersection relation. This research is, in part, an attempt to clarify this structure.
} 
onality between the notions of semantic class in the two resources" (Jones and Onyshkevych, 1996).

Dorr (To appear) reports more specifically on the percentage of errors and omissions of this type in a related experiment involving WordNet and LDOCE. This research cites a $61 \%$ correlation between syntactic codes and Levin classes, with $22 \%$ of the cases falling under syntactic omissions and only $17 \%$ pertaining to orthogonality between WordNet and Levin.

Saint-Dizier (1996) suggests that there is indeed orthogonality between syntax and semantic verb classes. According to the results of his experiments on French, verb classes formed on a strict syntactic basis "do not exhibit a very high rate of semantic relatedness (about 50\%)."

Palmer, Rosenzweig and Dang (1997) suggest that the appropriate granularity must be found for the mapping between these two types of resources to become clear. They derive 129 additional classes from Levin, using sets of classes that shared at least three verb tokens. The three-member threshold ensured systematic relations between classes, rather than spurious intersections due to homonymy or polysemy. This prevented e.g., draw (water) and draw (a picture) from creating an intersective class (Rosenzweig and Dang, 1997). They used these intersective sets, hand-tagged with WordNet senses, to investigate the relationship between WordNet and Levin for two classes: 21.1 Cut verbs and 23.2 Split verbs. They determined that the intersective sets, augmented by verbs sharing the WordNet semantics, created an extensional hierarchy that mirrored the synonym set nodes in Levin.

In this paper, we report on several experiments to derive the intersective sets based on word senses rather than tokens. We describe our experiments and compare the results with the classes in Rosenzweig, et al. (1997), and examine the results of the intersection on the classes described in Palmer, Rosenzweig, and Dang. We use the syntactic properties of the intersective sets to evaluate the polysemy reduction of Palmer, et al. (1997). Our future work will involve an exploration into the mapping of WordNet and Levin's classes on a large scale in collaboration with Rosenzweig, Palmer and Dang.

\section{Experiments}

In these experiments we draw upon a hand-crafted database of Levin verbs with WordNet sense tags created for automatic lexicon acquisition at the University of Maryland (Dorr and Jones, 1996b; Dorr and Jones, 1996a; Dorr and Jones, 1996c; Dorr, 1997). Levin verbs were tagged by hand with a set of WordNet senses, presented to the user as a set of logical addresses (e.g., 1-7) which are converted internally into WordNet addresses (e.g., 00416048-00416054). This process took a single person-month, with the aid of an interface for typing in human semantic judgments on the words in context. Each verb has between 1 
and 9 senses, with an average of 2.5 senses per word. ${ }^{2}$ We ran four experiments which we describe below, detailing examples from a small set of classes and comparing them with the results of Rosenzweig, et. al.

Experiment 1: We generated a candidate set of 616 intersective classes that included at least one word-sense pair in both classes. Each word-sense counted as a potential candidate in this experiment, even if other senses for the same word were not in both Levin classes. As problems of homonymy and polysemy were avoided by sense-tagging, we did not require a three-member threshold: 62 class-pairs overlapped those of the experiment of Rosenzweig et al., while 554 were unique to our experiment. Rosenzweig et al. had 58 additional class-pairs. ${ }^{3}$

\begin{tabular}{|c|c|c|}
\hline Only in Experiment 1 & Common Pairs & Only in Rosenzweig \\
\hline 554 & 62 & 58 \\
\hline
\end{tabular}

Examples of each case are given here:

- Common Pairs

The classes 10.1 (Remove Verbs) and 10.5 (Steal Verbs) are intersective in Rosenzweig's experiment, as there are three words in common: extract, winkle and withdraw. This pair is also included by our experiment 1 since classes 10.1 and 10.5 share at least one: all in this case. Another example of a case where our results overlapped with those of Rosenzweig, et al. is the combination of class 26.5 (Knead Verbs) and 45.4 (Other Alternating Verbs of Change of State). We had the following output:

26.5 and 45.4 :

collect_[1,3], compress_[1,2] freeze_4, melt_[1,2]

\section{- Only in Experiment 1}

The classes 10.1 and 10.6 (Cheat Verbs) are considered intersective only in our experiment 1, as there is only one overlapping sense of $c u l l$ between the two. Rosenzweig et al.'s threshold excluded this pair, to eliminate possible problems of polysemy and homonymy. Further examination is necessary to determine the extent to which cull as a Verb of Removal (10.1) has the same meaning as cull as a Possessional Deprivation (Cheat) Verb (10.6). We detail a similar examination in $3 .^{4}$

\footnotetext{
${ }^{2} 3991$ of Levin's 4183 verbs are annotated, with 6507 predicates indicating verb-sense membership in a specific Levin Class and words not in WordNet excluded.

${ }^{3}$ It is interesting to note that experiments 1 and 3 produce almost exactly the same overlap with the results of Rosenzweig et al., with the exception of one pair in experiment 1 that doesn't occur in experiment 3. This provides additional support for the hand-tagged results of Rosenzweig et al.

${ }^{4}$ In addition, 10.1 and 10.6 differ on the locative alternation: they each allow only one form and not the other, in mutually exclusive distribution. It is not clear how Levin expects cull to behave in this case.
} 


\section{- Only in Rosenzweig}

Classes 10.5 and 13.5.2 (Obtain Verbs) share a total of seven verbs: cadge, grab, recover, regain, retrieve, seize and snatch, making this a strong candidate for Rosenzweig et al.'s intersective classes. While there does seem to be some semantic correlation between the verbs in 'obtaining' and 'stealing,' there seems to be some difference in the connotation of the verbs in these two classes. As such, the same verb tokens received different senses in our hand-tagging depending on the class. Verbs in 13.5.2 imply a basic action, while the verbs in 10.5 connote the idea that the action is illegal. This difference was enough to cause all verbs involved to have different senses, and thus this pairing was rejected as in intersective class in our experiment.

Experiment 2: In this experiment we included only those class pairs matching all senses of a given word in the two classes. Thus, the criterion for class-pair candidacy was that the two classes in question share all of each others' WordNet senses for at least one verb-token. This experiment reduced the candidate set to 244; 42 of these class-pairs overlapped those of the Rosenzweig experiment, while 202 were unique to our experiment. Rosenzweig had 78 additional classpairs beyond those which overlapped with experiment 2. Of these, 58 matched no senses and 20 matched only some of the senses of any given token (these cases were included by experiment 1$).^{5}$

\begin{tabular}{|c|c|c|}
\hline Only in Experiment 2 & Common Pairs & Only in Rosenzweig \\
\hline 202 & 42 & 78 \\
\hline
\end{tabular}

Examples of each case are given here:

\section{- Common Pairs}

Again, classes 10.1 (Remove Verbs) and 10.5 (Steal Verbs) are considered intersective by Rosenzweig et al.'s experiment, as there are three words in common: extract, winkle and withdraw. This pair is included in our experiment 2, as classes 10.1 and 10.5 share all of the same senses of these words.

Recall from Experiment 1 that our overlapping set included 4 verbs for classes 26.5 and 45.4 . When we eliminated those verbs with mismatched senses, the intersective class contained only collect, compress:

\section{5 and 45.4 :}

$$
\text { collect_[1,3] compress_[1,2] }
$$

\footnotetext{
${ }^{5}$ Four of the pairs generated by Rosenzweig used an alphabetic suffix which was not part of the standard Levin classification, and thus not in our database. There were two pairs recognized as intersective by Rosenzweig et al.'s experiment but not by our algorithm because of missing verbs in our database (the verbs in question are actually not a part of WordNet). For example, we are missing the verbs hardboil and softboil, which Rosenzweig uses for classes 26.3 and 45.3. In every overlapping pair, all verbs in each class were present in our database.
} 
The verb freeze was one of the two verbs that was eliminated since it was tagged with sense 4 in 26.5 and 45.4 , but class 45.4 also contains senses 2 and 6 for this verb.

The intersection of 26.1 Build Verbs and 26.3 Verbs of Preparing illustrates an intersection with fewer verbs than Rosenzweig, et al., on principled reasons. They had: bake, cook, roll. We had bake_[1,2] and cook_[1, 2,3]. We excluded roll, since both classes shared sense 3 (00801140), but 26.3 also has sense 9 (00196539), associated with synsets that do not denote building, although they do indicate preparing, specifically 00196539: roll_9, seethe_5 and 00801140: flatten_with_a_roller_1, roll_3, roll_out_1, spread_with_a_roller_1.

\section{- Only in Experiment 2}

As in the experiment 1 comparison, the classes 10.1 and 10.6 (Cheat Verbs) are considered intersective only in our experiment 2, as cull only has one WordNet-sense in either class, and this one sense is the same in both classes. If cull had more than one sense in a given class, then all of these senses and only these senses would need to be included in a potential partner class. As stated before, Rosenzweig et al.'s results did not include this pair, as possible problems of polysemy and homonymy prevented total certainty that this was a true semantic overlap.

\section{- Only in Rosenzweig}

Classes 10.4.1 (The Manner subclass of Wipe Verbs) and 41.1.1 (Dress Verbs) share shave, strip and wash. However, shave and strip share no common senses between the two classes, and the classes only share sense 2 of wash, while 10.4.1 also includes senses 1 and 3 .

Experiment 3: We generated a candidate set of 956 intersective classes that shared at least one WordNet sense ("synset" numbers), whether or not that sense was attached to the same word in both Levin classes. The criterion for candidacy was then that two classes share at least one synset. All but one of these pairs in Experiment 1 is caught by this test, since the list of the sense numbers were converted to a list of synsets, independent of their associated verb tokens. 953 class-pairs fulfilled the requirements for candidacy, 63 of which overlapped those of the Rosenzweig et al.'s experiment, while 893 were unique to our experiment. Rosenzweig et al. had 57 additional class-pairs beyond those which overlapped with experiment 2.

\begin{tabular}{|c|c|c|}
\hline Only in Experiment 3 & Common Pairs & Only in Rosenzweig \\
\hline 893 & 63 & 57 \\
\hline
\end{tabular}

\section{- Common Pairs}

Yet again, the classes 10.1 (Remove Verbs) and 10.5 (Steal Verbs) are captured by Rosenzweig's experiment, as there are three words in common: 
extract, winkle and withdraw. This pair is also included in our experiment 3 (as are all pairs found by experiment one), as classes 10.1 and 10.5 share all of the same word-senses, and therefore all of the same WordNet synsets. Only one WordNet synset is enough however, and it needn't come from the same word in each class.

- Only in Experiment 3

Classes 10.1 and 31.1 (amuse verbs) do not actually share any words, and as such would never be selected by Rosenzweig's experiment. The only thing they share is the WordNet synset 1395808, derived from ostracize in 10.1 and cut in 31.1, which is enough for inclusion in this set. Add "Other elements in this set include..."? [find elements, Dav?] comment from previous version...(add other elements in synset, to see if there is some sense in our positing this set.

\section{- Only in Rosenzweig}

Again, classes 10.5 and 13.5.2 (Obtain Verbs) are not included in our experiment, but were captured by Rosenzweig. It is interesting to note that although experiment three resulted in an almost $50 \%$ increase in size, only one additional word from Rosenzweig's experiment was accounted for. As stated before, the seven verbs cadge, grab, recover, regain, retrieve, seize and snatch made this a strong candidate for Rosenzweig. The differences in these two classes were apparently strong enough, however, to completely block the sort of fine grained semantic overlap this experiment categorizes.

Experiment 4: We generated a single candidate pair in which all of the synsets in a given class were also found in another class. The synsets of Rushing (53.2) are a proper subset of the 133-member Run-class synsets (51.3.2). Example verbs from these synsets include hasten_ $[1,2,3]$, hurry_ $[1,2,3]$ and rush_[1,3,4]. Interestingly, the alternation properties noted by Levin also have a subset relation, though not transparently so. The only property given for the Verbs of Rushing is the fact that all can be used transitively in the causative construction (Her sister hurried. Maggie hurried her sister.). Among other properties, the Run verbs participate in the Induced Action Alternation, a type of causative (Levin, 1993, p. 31).

This suggests that the Verbs of Rushing are a subclass of the Run verbs that is semantically rather than syntactically described: the Verbs of Rushing "describe doing something quickly" and the Run verbs "describe the manners in which animate entities can move" (Levin, 1993, pp. 271, 267). The classes appear to differ only with respect to what Levin (and others) have called the semantic "constant", that aspect of verb meaning that is said to be linguistically inert, and not therefore part of the semantic "structure" (Grimshaw, 1993; Pinker, 1989; Levin and Rappaport Hovav, To appear).

Further examination of the intersective classes from these experiments will 
determine whether, in fact, some of the classes made by Levin are, in fact, semantically based in a way revealed by WordNet. In the next section we show that the case discussed by Palmer, et al. (1997) suggests a slightly different result: that the intersective semantic classes have a syntactic intersection as well. This would imply a somewhat different lexical organization than Palmer, et al. (1997).

\section{$3 \quad 21.1$ Cut and 23.2 Split verbs}

Palmer, et al. (1997) claim that the Levin classes do, in fact, reflect semantic distinctions made by WordNet. They suggest reflecting the Levin-WordNet mapping more directly by removing the type of polysemy encoded by the intersective classes. The Cut and Split classes derive the intersective set shown below:

(1) 21.1 Cut Verbs (Levin, 1993)

"The meaning of these verbs involves notions of motion, contact, and effect ... a "separation in material integrity.",

chip, clip, cut, hack, hew, saw, scrape, scratch, slash, snip

(2) 23.2 Split Verbs (Levin, 1993)

"In the use illustrated here, each of these verbs manifests an extended sense which might be paraphrased 'separate by $V$-ing,' where ' $V$ ' is the basic meaning of that verb."

blow, break, cut, draw, hack, hew, kick, knock, pry, pull, push, rip, roll, saw, shove, slip, tug, yank

(3) 21.1 and 23.2 (Rosenzweig and Dang, 1997)

cut, hack, hew, saw

The results of our experiments (specifically 3 below) suggest chop as a potential additional member:

- Experiment 1: any senses of a given word overlap 21.1 and 23.2: cut_1, hack-1, hew_1, hew_2, saw_1

- Experiment 2: all senses of a given word overlap 21.1 and 23.2: cut_1, hack-1, hew_1, hew_2, saw_1

- Experiment 3: any WordNet synsets overlapping 21.1 and 23.2: 00717504 (chop_4, hack_1), 00719888 (hew_1, hew_out_1), 00894185 (cut_1, separate_with_an_instrument_1), 00899031 (cut_with_a_saw_1, saw_1), 00719697 (hew_2, strike_with_an_axe_1)

Palmer, et al. observe that WordNet describes the following semantic classes: 
(4) Manner of cutting that results in separation into pieces:

chip, clip, cut, hack, hew, saw, slash, snip

(5) Manner of cutting that doesn't separate completely:

scrape, scratch

Following a suggestion by Levin (1993) p.166, Palmer, et al. (1997) suggest augmenting the Split class, and hence the second-order (intersective) class, to include chip, clip, slash, and snip. Augmenting the Levin classes in this way, they argue, reduces polysemy in the Levin classes and allows an extensional mapping between Levin and WordNet. Verbs derive their syntactic properties via inference from the Levin classes and their intersections and semantic properties through membership in the WordNet classes. The Levin classes could also be augmented by the WordNet verbs in experiment 3, e.g. chop.

However, the intersective relationship may, in fact, be more complicated, since the verbs in the two classes do not share all the syntactic properties cataloged by Levin. Consulting Levin, we find that the classes share one "alternation" (both allow the middle: Whole wheat bread cuts/splits easily.); however, only the Split verbs allow the causative (Carol cut/split the bread; The bread *cut/split). Levin lists no other alternations that reference both classes.

Because of the conflict in the causative, one would expect verbs in the two Levin classes may behave differently on the causative, depending on which sense is operative. However, whether or not the inchoative is good appears to depend on whether the event named could be conceived as internally caused (e.g. a metal button that cut itself loose). This element of meaning cross-cuts the classes in Levin and WordNet.

(6) (i) Mary cut her hair./*Her hair cut.

(ii) I cut the button and the vest apart./?The button and the vest cut apart.

Further examination of these (and other) intersective classes is necessary, enhanced by the augmented alternations database from Dorr and Olsen (1996). This database was produced by cross-referencing the classes in Part II Levin's book with the alternations she describes in Part I, yielding a greater intersection of syntactic properties for inspection, as given below (' + ' indicates that a verb class allows both forms of an alternation, 'A' that it allows the first form).

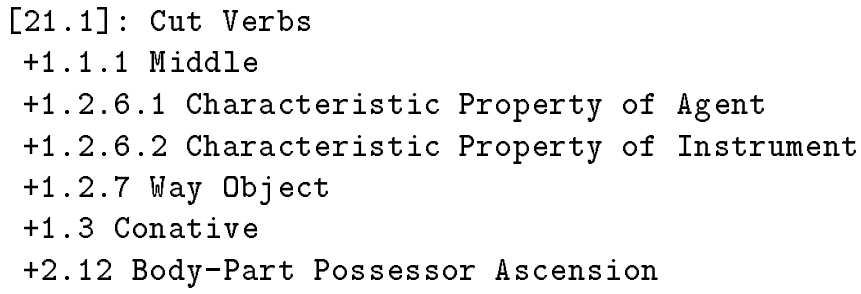




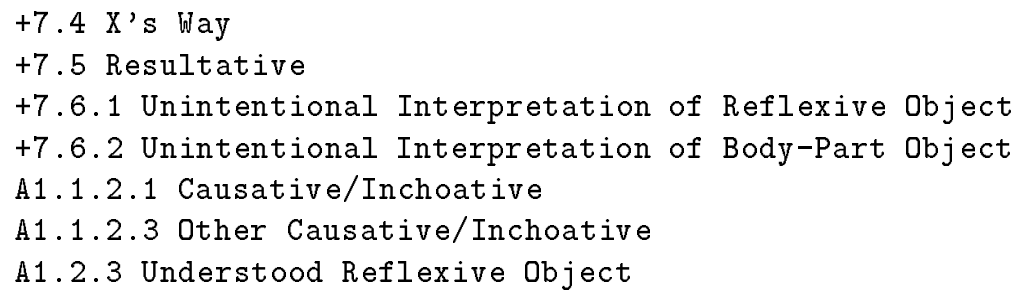

Note that the alternations shared by the classes are increased (including, in addition to the Middle, the Characteristic Property of Agent, Way Object, and the Conative), whereas no new conflicts are introduced. This provides further support for the intersective classes. The behavior of the verbs on the remaining properties that do not conflict (e.g. the Reciprocals that occur with the Split verbs but not the others) may provide other syntactic properties for investigation, permitting a teasing apart of the properties that distinguish these classes.

\section{Conclusions and Future Research}

We have described results of experiments using WordNet synset tags to evaluate the semantic properties of the verbs classes cataloged by Levin (1993). We minimized the impact of the non-exhaustiveness of Levin's database by adding intersective classes to the experiment and to provide a clearer picture of the relationship between WordNet/EuroWordNet and the semantic classes. This research is part of a larger effort to combine the resources of WordNet and EuroWordNet with the LCS interlingua, based on Levin classes, for machine translation and other NLP applications. 
Experiment 1 indicates that there are potentially a large number of candidates for intersective classes since WordNet is finely articulated. We expect this level of granularity to yield a large-scale mapping, but the quality and completeness of the classes is difficult to evaluate given the incompleteness of Levin. Experiment 2 illustrates that it is possible to pare down these fine-grained classes, with each intersective class based on fewer verbs, on principled reasons, than those of Rosenzweig, et al.'s experiment. Experiment 3 addresses the problem of incompleteness in Levin, i.e., it leads to the question of whether two semantically intersecting classes might not have the same words due to omissions in Levin. Additional experimentation would be needed to determine if the new intersecting classes are distinguished by additional syntactic characteristics not included in Levin's book. Experiment 4 suggests the possibility of collapsing the structure in Levin, itself (cf. results of work by Olsen, Dorr, and Thomas, this workshop).

We have examined the syntactic behavior of the verbs with respect to the newly postulated intersective class for the $C u t$ and Split verbs. The results of this investigation provide further support for the intersective classes and for the possibility of arguing for or against such classes on syntactic grounds.

\section{Acknowledgments}

The work was supported, in part, by National Science Foundation Presidential Faculty Fellowship (PFF/PECASE) Award IRI-9629108, Department of Defense contract MDA90496C1250, DARPA/ITO Contract N66001-97-C-8540, Army Research Laboratory contract LETTER 11097 through United Research Corporation, and Army Research Laboratory contract DAAL03-91-C-0034 through Battelle.

\section{References}

Calzolari, Nicoletta, Antonia Marti, Horacio Rodriguez, Felisa Verdejo, Piek Vossen, and Yorick Wilks. To appear. EuroWordNet Project (Title under Revision). Computers and the Humanities.

Dorr, Bonnie J. 1997. Large-Scale Acquisition of LCS-Based Lexicons for Foreign Language Tutoring. In Proceedings of the ACL Fifth Conference on Applied Natural Language Processing (ANLP), pages 139-146, Washington, DC.

Dorr, Bonnie J. To appear. Large-Scale Dictionary Construction for Foreign Language Tutoring and Interlingual Machine Translation. Machine Translation, 12(1). 
Dorr, Bonnie J. and Douglas Jones. 1996a. Acquisition of Semantic Lexicons: Using Word Sense Disambiguation to Improve Precision. In Proceedings of the Workshop on Breadth and Depth of Semantic Lexicons, 34th Annual Conference of the Association for Computational Linguistics, pages 42-50, Santa Cruz, CA.

Dorr, Bonnie J. and Douglas Jones. 1996b. Robust Lexical Acquisition: Word Sense Disambiguation to Increase Recall and Precision. Technical report, University of Maryland, College Park, MD. Submitted to Computational Linguistics.

Dorr, Bonnie J. and Douglas Jones. 1996c. Use of Syntactic and Semantic Filters for Lexical Acquisition: Using WordNet to Increase Precision. In Proceedings of the Workshop on Predicative Forms in Natural Language and Lexical Knowledge Bases, pages 81-88, Toulouse, France.

Dorr, Bonnie J. and Mari Broman Olsen. 1996. Multilingual Generation: The Role of Telicity in Lexical Choice and Syntactic Realization. Machine Translation, 11(1-3):37-74.

Grimshaw, Jane. 1993. Semantic Structure and Semantic Content in Lexical Representation. unpublished ms., Rutgers University, New Brunswick, NJ.

Jones, Doug and Boyan Onyshkevych. 1996. Evaluating Levin Classes vs Wordnet. Technical report, Department of Defense, Washington, D.C., November 19.

Levin, Beth. 1993. English Verb Classes and Alternations: A Preliminary Investigation. University of Chicago Press, Chicago, IL.

Levin, Beth and Malka Rappaport Hovav. To appear. Building Verb Meanings. In M. Butt and W. Gauder, editors, The Projection of Arguments: Lexical and Syntactic Constraints. CSLI.

Miller, George A. 1986. Dictionaries in the Mind. Language and Cognitive Processes, 1:171-185.

Miller, George A. 1990. WordNet: An On-Line Lexical Database. International Journal of Lexicography, 3:235-312.

Miller, George A. and Christiane Fellbaum. 1991. Semantic Networks of English. In Beth Levin and Steven Pinker, editors, Lexical and Conceptual Semantics, Cognition Special Issue. Elsevier Science Publishers, B.V., Amsterdam, The Netherlands, pages 197-229.

Palmer, Martha, Joseph Rosenzweig, and Hoa Trang Dang. 1997. Intersective Levin Classes. In Workshop on Tagging Text with Lexical Semantics: Why, What, and How?, Washington, D.C., April 4-5. Presentation at the Working Group on Combining Knowledge Sources for Automatic Semantic Tagging. 
Pinker, Steven. 1989. Learnability and Cognition: The Acquisition of Argument Structure. The MIT Press, Cambridge, MA.

Rosenzweig, Joseph and Hoa Trang Dang. 1997. Intersective Levin Classes, March. Presentation at the Verb Working Group, University of Pennsylvania.

Saint-Dizier, Patrick. 1996. Semantic Verb Classes Based on 'Alternations' and on WordNet-like Semantic Criteria: A Powerful Convergence. In Proceedings of the Workshop on Predicative Forms in Natural Language and Lexical Knowledge Bases, pages 62-70, Toulouse, France. 